\title{
ON TRANSFORMATIONS OF THE 3-SPHERE FIXING A KNOT
}

\author{
BY CHARLES H. GIFFEN ${ }^{1}$
}

Communicated by P. E. Conner, Jr., May 25, 1967

The Smith conjecture (after P. A. Smith [1]) is that no tame, knotted, simple closed curve in the 3 -sphere is the fixed point set of a periodic transformation of the 3 -sphere. That is to say, for any nontrivial tame knot $\left(S^{3}, K\right)$, the group $F\left(S^{3}, K\right)$ of orientation preserving autohomeomorphisms of $S^{3}$ which are the identity on $K$ has no elements of finite order.

The purpose of this paper is to show that, except for the trivial type of knot, the identity path component of $F\left(S^{3}, K\right)$ under the compact-open topology has no elements of finite order. Hence for nontrivial knots, if $F\left(S^{3}, K\right)$ has elements of order $r>1$, then $\pi_{0}\left(F\left(S^{3}, K\right)\right)$ has elements of order $r$.

This suggests a strong Smith conjecture that $\pi_{0}\left(F\left(S^{3}, K\right)\right)$ has no elements of finite order. The reduction of this strong Smith conjecture to an algebraic Smith conjecture about certain automorphisms of the group system of a knot will be the subject of another paper on homeotopy groups of knots.

Indeed, the strong Smith conjecture for torus knots and others may be verified in this manner. This supersedes the special proof of the Smith conjecture for torus knots given by the author in [2]. R. H. Fox [3] has obtained an elementary proof of the Smith conjecture for torus knots and a different, somewhat complicated algebraic interpretation of the Smith conjecture for knots whose knot groups are centerless. The relation of Fox's results with ours will be discussed in the paper on homeotopy groups of knots.

Let $\left(S^{3}, K\right)$ be a nontrivial tame knot, and let

$$
h:\left(S^{3}, K\right) \rightarrow\left(S^{3}, K\right)
$$

be a homeomorphism of pairs such that $h \mid K=1, h^{r}=1$ for some integer $r>1$, and $h$ is isotopic to the identity (written $h \approx 1$ ) via an isotopy

$$
H:\left(S^{3}, K\right) \times I \rightarrow\left(S^{3}, K\right) \times I
$$

(i.e. $H$ is a homeomorphism such that $p_{I} H=p_{I}$ where $p_{I}$ denotes the projection of $\left(S^{3}, K\right) \times I$ onto $\left.I\right)$ such that $H \mid K \times I=1$. The main theorem is the following.

1 This research was supported by National Science Foundation grant GP-2440. 
THEOREM. Under the above conditions, $h=1$, provided $\left(S^{3}, K\right)$ is a $\dot{k}$ not such that no power of a meridian of $K$ lies in the center of the knot group $G=\pi_{1}\left(S^{3}-K\right)$.

Proof. Since $h \approx 1$ via the isotopy $H$, then $h^{r}=1 \approx 1$ via the isotopy $H^{r}$, and $H^{r} \mid K \times I=1$. Let $o \in S^{3}-K$ be a base point, and let $P: I \rightarrow S^{3}-K$ be the path defined by $H^{r}(o, t)=(P(t), t)$. Then $P$ is actually a loop and hence represents an element $z$ of $G=\pi_{1}\left(S^{3}-K, o\right)$. With $o$ chosen very close to $K$, it is easy to see that $z=m^{k r+1}$, where $m$ is the (homotopy) class of some meridian of $K$ and $k$ is some integer, if we assume $h \neq 1$.

Moreover, $z$ is in the center of $G$ since it is the class of the connecting path $P$ of an isotopy of 1 with itself. But this contradicts the hypothesis of the theorem. Hence $h=1$.

REMARK. If $\left(S^{3}, K\right)$ is a torus knot of type $(p, q)$, then the center of $G$ is generated by $\mu^{p q} \lambda$, where $(\mu, \lambda)$ is a (suitably oriented) meridianlongitude pair for $K$.

This is seen by looking at the linking number of the components of the torus link of type $(2 p, 2 q)$, which is $p q$, and observing that one component generates the center of the knot group of the other component and parallels it. Hence the following holds.

Corollary. If $\left(S^{3}, K\right)$ is a torus knot, then the identity component of $F\left(S^{3}, K\right)$ has no elements of finite order.

It is a conjecture of L. Neuwirth [4] that only knot groups of torus knots have centers. Zieschang and Burde have recently proved this conjecture about torus knots, hence the Theorem and Corollary give the result stated in the second paragraph.

\section{REFERENCES}

1. P. A. Smith, Transformations of finite period. II, Ann. of Math. (2) 40 (1939), 690-711.

2. C. H. Giffen, Fibered knots and periodic transformations, Ph.D. Thesis, Princeton University, Princeton, N. J., 1964.

3. R. H. Fox, Two theorems about periodic transformations of the 3-sphere, Mich. Math. J. 14 (1967), 331-334.

4. L. Neuwirth, Knot groups, Ann. of Math. Studies, no. 56, Princeton Univ. Press, Princeton, N. J., 1965.

5. H. Zieschang and G. Burde, Eine Kennzeichnung der Torusknoten, Math. Z. 167 (1966), 169-176.

The Institute for Advanced Study and

The UNIVERSITY OF Virginia 